\title{
ISOLATED LEFT VENTRICULAR MYOCYTE CONTRACTILITY IN PATIENTS UNDERGOING CARDIAC OPERATIONS
}

R. Brent New, MD

James L. Zellner, MD

Latha Hebbar, MD

Rupak Mukherjee, PhD

Angela C. Sampson, BA

Jennifer W. Hendrick, BS

John R. Handy, MD,

Fred A. Crawford, Jr., MD

Francis G. Spinale, MD, $\mathrm{PhD}$

\begin{abstract}
Background: Because of methods required for obtaining isolated left ventricular myocytes, evaluation of the contractile function of isolated left ventricular myocytes in normal human patients has been limited. Accordingly, the goal of the present study was to develop a means to isolate human left ventricular myocytes from small myocardial biopsy specimens collected from patients undergoing elective coronary artery bypass operations and to characterize indices of myocyte contractile performance. Methods: Myocardial biopsy specimens were obtained from the anterior left ventricular free wall of 22 patients undergoing coronary artery bypass operations. Myocytes were isolated from these myocardial samples by means of a stepwise enzymatic digestion method and micro-trituration techniques. Isolated left ventricular myocyte contractile function was assessed by computer-assisted high-speed videomicroscopy under basal conditions and in response to $\beta$-adrenergic receptor stimulation with isoproterenol. Results: A total of 804 viable left ventricular myocytes were successfully examined from all of the myocardial biopsy specimens with an average of $37 \pm 4$ myocytes per patient. All myocytes contracted homogeneously at a field stimulation of 1 $\mathrm{Hz}$ with an average percent shortening of $3.7 \% \pm 0.1 \%$ and shortening velocity of $51.3 \pm 1.3 \mu \mathrm{m} / \mathrm{s}$. After $\beta$-adrenergic receptor stimulation with isoproterenol, percent shortening and shortening velocity increased 149\% and $118 \%$ above baseline, respectively $(P<.05)$. Conclusion: The unique results of the present study demonstrated that a high yield of myocytes could be obtained from human left ventricular biopsy specimens taken during cardiac operations. These myocytes exhibited stable contractile performance and maintained the capacity to respond to an inotropic stimulus. The methods described herein provide a basis by which future studies could investigate intrinsic and extrinsic influences on left ventricular myocyte contractility in human beings. (J Thorac Cardiovasc Surg 1998;116:495-502)
\end{abstract}

T: he determinants of left ventricular (LV) pump function are preload, afterload, and intrinsic contractile state. Because of the interactions of

From the Division of Cardiothoracic Surgery and Department of Anesthesiology, Medical University of South Carolina, Charleston.

Supported by a National American Heart Association Grant and National Institute of Health grant HL-56603. F.G.S. is an Established Investigator of the American Heart Association.

Received for publication Dec 9, 1997; initial revisions requested Jan 30, 1998; final revisions received May 22, 1998; accepted for publication May 22, 1998.

Address for reprints: Francis G. Spinale, MD, PhD, Cardiothoracic Surgery, Room 418 CSB, Medical University of South Carolina, Charleston, SC 29425.

Copyright (C) 1998 by Mosby, Inc.

$0022-5223 / 98 \$ 5.00+0 \quad \mathbf{1 2} / \mathbf{1} / 91983$ these determinants, assessment of LV myocardial contractile performance in vivo can be problematic. Since the fundamental contractile unit of the LV is the myocyte, a large number of studies have used isolated myocytes from various animal species to evaluate contractile function and excitation-contraction processes. ${ }^{1-5}$ To obtain LV myocytes, the vast majority of these past studies used isolation techniques in which large portions of myocardium were digested through coronary perfusion of a collagenase solution. ${ }^{1-6}$ In light of these coronary perfusion requirements, isolated human LV myocyte studies have been restricted to myopathic hearts explanted at the time of transplantation or the uncommon situation of harvested hearts deemed unsuitable for transplantation. ${ }^{7-11}$ More recently, it has been suggested that human LV myocytes can be obtained 


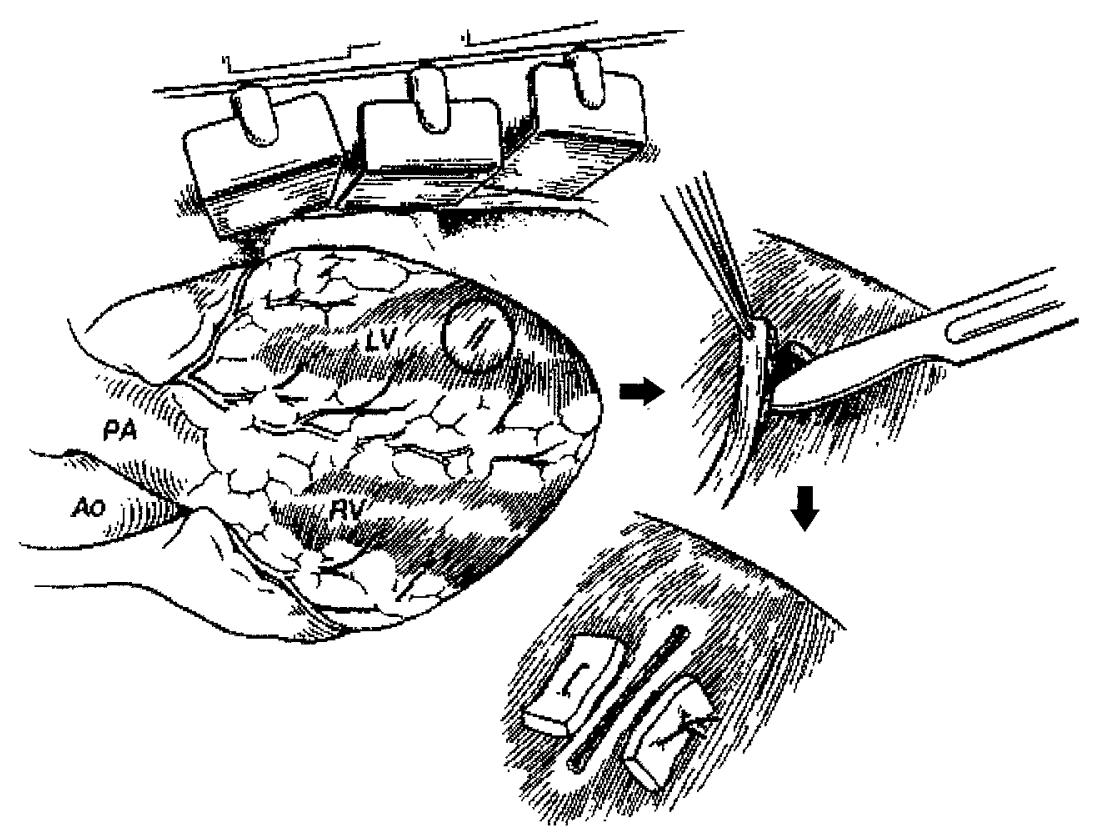

Fig 1. LV myocardial biopsy procedure including location of the biopsy site, excision of the specimen, and closure of the site with pledget-supported Prolene mattress sutures. $A o$, Aorta; $P A$, pulmonary artery; $R V$, right ventricle; $L V$, left ventricle.

from small myocardial biopsy specimens. ${ }^{12}$ Previous reports have used these human $\mathrm{LV}$ myocyte preparations to examine cellular electrophysiology and calcium transients. ${ }^{7,8,11,12}$ However, limited data exist with respect to contractile performance of human LV myocytes.9, 10,13 Furthermore, to what extent the LV biopsy and isolation technique can be used in patients undergoing cardiac operations remains unclear. Accordingly, the goal of the present study was to characterize indices of isolated LV myocyte contractile performance in adults undergoing cardiac surgery.

\section{Methods}

In the present study, LV myocardial biopsy specimens were obtained from patients undergoing coronary artery bypass operations. Myocytes were isolated from these myocardial samples by means of a modification of a previously described method. ${ }^{12}$ Isolated LV myocyte contractile function was assessed by computer-assisted highspeed videomicroscopy ${ }^{14}$ under basal conditions and in response to inotropic stimulation with isoproterenol.

Biopsy procedure. The study protocol was reviewed and approved by the Institutional Review Board of the Medical University of South Carolina before any patient recruitment. Patients scheduled to undergo elective coronary artery bypass operations were selected for the study, and informed written consent was obtained. The mean patient age was $62 \pm 2$ years with a range of 47 to 76 years. Preoperative LV function was assessed by ventriculogra- phy or echocardiography. These imaging modalities were used to confirm that no abnormal wall motion was present in the anterior $\mathrm{LV}$ rcgion. $\mathrm{LV}$ ejection fraction was $55 \%$ $\pm 2 \%$ with a range of $30 \%$ to $70 \%$. After induction of general anesthesia with sufentanil and isoflurane, a median sternotomy was performed. During the time the heart was prepared for cardiopulmonary bypass, the anterior LV was inspected for areas of infarction, fibrosis, or regional wall motion abnormalities. The region devoid of apparent epicardial vessels and fat along the distal left antcrior descending coronary artery, triangulated by the LV apex and the last diagonal branch, was targeted for biopsy. With the use of a No. 11 scalpel, a small biopsy specimen (averaging $5 \times 4 \mathrm{~mm}$ ) was excised from the LV epicardial surface to a depth of approximately $3 \mathrm{~mm}$. The specimen was immediately placed in an ice cold oxygenated transfer solution as described in the next section. The biopsy site was closed with pledget-supported 3-0 Prolene sutures (Ethicon, Inc., Somerville, NJ). An illustration of the biopsy procedure is provided in Fig 1.

Myocyte isolation. The LV myocardial biopsy specimens were immediately placed in ice cold oxygenated HEPES-buffered balanced salt solution ( $\mathrm{pH} 7.4$ ) containing the following: glucose, $10 \mathrm{mmol} / \mathrm{L}$; taurine, 20 $\mathrm{mmol} / \mathrm{L}$; creatine, $5 \mathrm{mmol} / \mathrm{L}$; succinate, $5 \mathrm{mmol} / \mathrm{L}$; calcium $\left(\mathrm{Ca}^{++}\right) 2 \mu \mathrm{mol} / \mathrm{L}$; nirriloacetic acid, $5 \mathrm{mmol} / \mathrm{L} ;$ and 2,3-butanedione monoxime (BDM [Sigma Chemical Corp, St Louis, Mol), $15 \mathrm{mmol} / \mathrm{L}$. The specimens were transported to the laboratory within 30 minutes. While the specimens were maintained in solution, they were dissected free of any fatty tissue and finely minced. Specimens were then transferred to another oxygenated 
HEPES solution containing $\mathrm{Ca}^{++}(50 \mu \mathrm{mol} / \mathrm{L})$, protease (4 U/mL; Sigma Type XXIV, Sigma Chemical Corp), collagenase $(400 \mathrm{U} / \mathrm{mL}$; Worthington Biochemical Corp, Freehold, NJ), and BDM $(15 \mathrm{mmol} / \mathrm{L})$ and allowed to gently bubble in a water jacket at $35^{\circ} \mathrm{C}$ for 30 minutes. The myocytes were then resuspended in an oxygenated collagenase solution containing hyaluronidase $(0.5 \mathrm{mg} /$ $\mathrm{mL}$, Sigma Chemical Corp) and gently agitated for 90 minutes at $35^{\circ} \mathrm{C}$. The concentrations of enzymes (protease, collagenase, and hyaluronidase) used in the isolation procedure were based on previously published studies, $1,7,12,13$ as well as preliminary studies by this laboratory, to maximize cell yields. Myocytes were then allowed to settle 3 to 5 minutes and form a pellet at room temperature. The supernatant was discarded and myocytes were resuspended in an oxygenated HEPES solution containing aerobic substrates, a $0.5 \mathrm{~g} / \mathrm{L}$ concentration of bovine serum albumin (Sigma Chemical Corp) and a 300 $\mu \mathrm{mol} / \mathrm{L}$ concentration of $\mathrm{Ca}^{++}$. This last step was repeated to wash out any remaining BDM. A large number of quiescent myocytes were routinely obtained (10\%-50\% yields) with yields often greater than $50 \%$. BDM, which reversibly inhibits $\mathrm{Ca}^{+1}$-induced force development by myofilaments, in addition to a zero or low $\mathrm{Ca}^{++}$period, has been shown to produce higher cell yields by reducing cutting injury and hypercontracture injury early during the myocyte isolation process. ${ }^{15,16}$ Myocytes were stored at room temperature in this final solution until used for contractility analysis.

Myocyte contractility measurements. Isolated myocytes were placed into a thermostatically controlled chamber containing an oxygenated Krebs balanced salt solution (pH 7.4) containing dextrose and $\mathrm{Ca}^{++}(1 \mathrm{mmol} / \mathrm{L})$ at $37^{\circ} \mathrm{C}$ and imaged with an inverted microscope (World Precision Instruments, Sarasota, Fla). Individual myocytes were selected for analysis on the basis of a characteristic rod-shaped appearance with no membrane blebbing and quiescence in the presence of extracellular $\mathrm{Ca}^{++}(1$ $\mathrm{mmol} / \mathrm{L}$ ). A homogeneous contractile response was elicited in each myocyte by field stimulation of the chamber at $1 \mathrm{~Hz}$ (S11, Grass Instruments, Quincy, Mass) with a 5-ms pulsc width and voltages $10 \%$ above contraction threshold. The polarity of the stimulating electrodes was alternated at every pulse to prevent buildup of electrochemical by-products. ${ }^{14}$ Myocyte contractions were imaged with the use of a charge-coupled device with a non-interlaced scan rate of $240 \mathrm{~Hz}$ (GPCD60, Panasonic, Secaucus, NJ). In a past report from this laboratory, Fourier analysis of the myocyte contraction data revealed that $99 \%$ of the power spectrum component of the contractile signal was contained within $8 \mathrm{~Hz}{ }^{14}$ Thus, according to the Nyquist sampling thcorem, a sampling frequency of $16 \mathrm{~Hz}$ would be the minimum required to digitize the myocyte contractile signal. Therefore the digitization sampling frequency of $240 \mathrm{~Hz}$ that was uscd in the present study was adequate to accurately characterize the myocyte contractile signal. Myocyte motion signals were captured with the cell parallel to the video raster lines, and this video signal was input through an edge detector system (Crescent Electronics, Sandy, Utah). The changes in light intensity at the myocyte edges were used to track myocyte motion. The distance between the left and right myocyte edges was converted into a voltage signal, digitized, and input into a computer (80486; Zeos International, Minneapolis, Minn) for subsequent analysis, as previously described. ${ }^{17}$ Although myocyte contractility measurements were performed in this study within a 6 -hour period, myocytes did remain viable for up to 24 hours after isolation. Baseline contraction profiles for each selected myocyte were recorded from a minimum of 20 consecutive contractions. After baseline measurements wcre recorded, a random subset of myocytes was exposed to the $\beta$-adrenergic receptor agonist isoproterenol. After a 3-minute exposure to isoproterenol, contractions were recorded. Parameters computed from the digitized contraction profiles of all myocytes included percent shortening, velocity of shortening (micrometers per second), velocity of relengthening (micrometers per second), total contraction duration (milliseconds), and time to peak contraction (milliseconds). All parameters were calculated for each contraction and the results averaged for all contractions observed.

Data analysis. Myocyte function was analyzed patient to patient, with each patient being considered a separate block and the number of myocytes being considered repeated observations within each block. In addition, summary statistics were calculated in which all myocytes were combined for each patient. The myocyte contractile response to isoproterenol was compared to baseline values by means of a paired $t$ test. Myocyte contractile response to the different isoproterenol doses was compared by means of an unpaired $t$ test. Linear and nonlinear regression analysis was performed to determine the relationship between $L V$ ejection fraction and indices of LV myocyte contractile function. All statistical analysis was performed with the use of BMDP statistical software programs (BMDP Statistical Software, Inc, Los Angeles, Calif). Results are presented as mean \pm standard error of the mean unless otherwise indicated.

\section{Results}

Myocardial biopsy specimens were successfully collected from all 22 patients enrolled in the study. There were no complications related to the biopsy procedure. Of the study patients, $18 \%$ were taking angiotensin-converting enzyme inhibitors, 68\% $\beta$-adrenergic receptor antagonists, 36\% calcium channel antagonists, $9 \%$ digoxin, and $18 \%$ diuretics.

Viable LV myocytes were successfully obtained from all of the myocardial biopsy specimens and an average of 37 (standard deviation 20) individual myocytes were examined from each biopsy with a range of 5 to 71 myocytes. A phase-contrast photomicrograph of a group of representative isolated LV myocytes and a representative contraction profile for a single myocyte are shown in Fig 2 . The actual values for each myocyte broken down for each patient are illustrated in Fig 3. Also, the mean values for each patient for resting length, percent shortening, and shortening velocity are included. A distribution profile of individual myocyte resting 

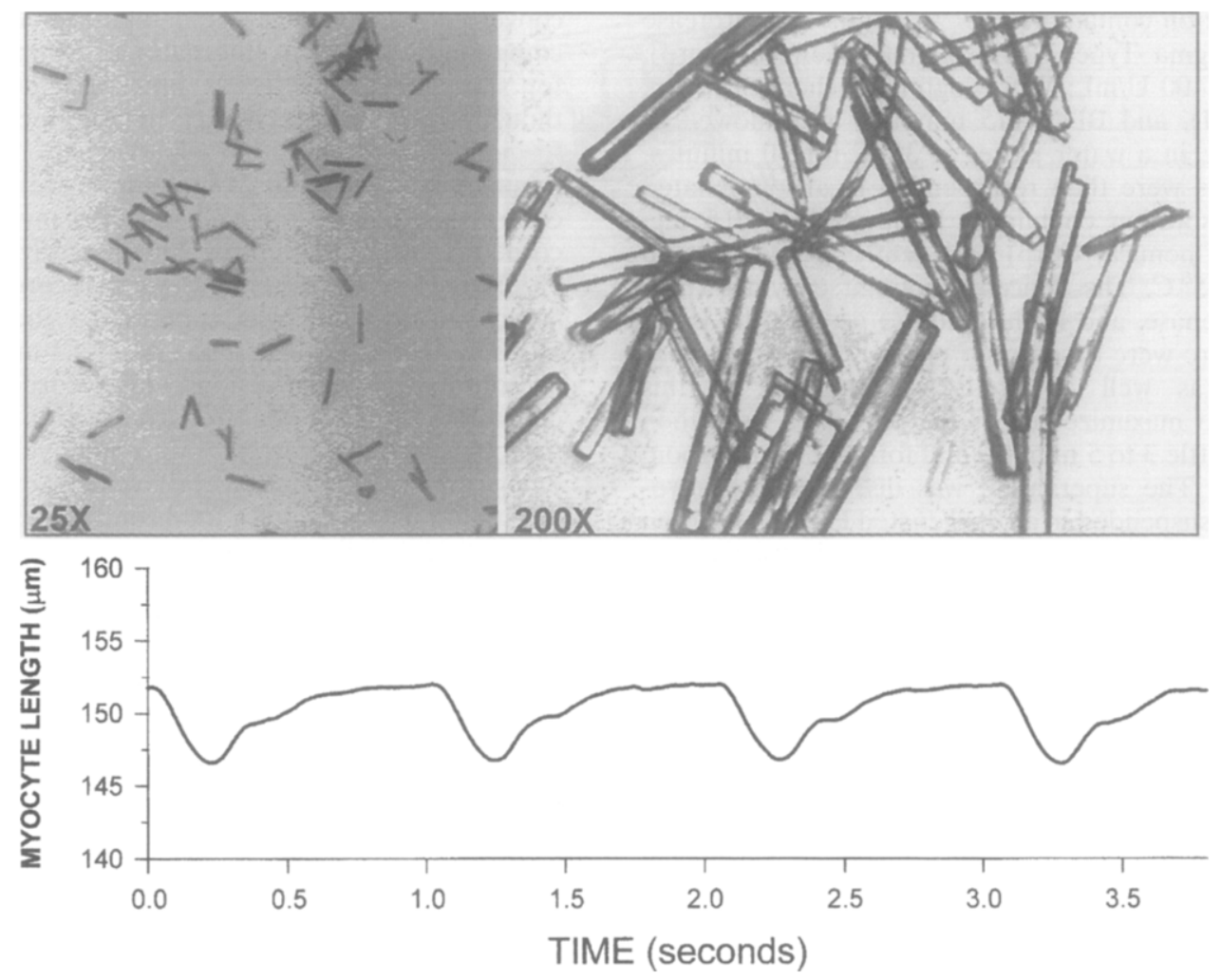

Fig 2. Top, Representative phase-contrast photomicrographs of LV myocytes obtained from a myocardial biopsy specimen viewed at low power $(25 \times)$ and at high power $(200 \times)$. After isolation, a high yield of viable myocytes maintained a characteristic rod-shaped appearance and were quiescent in extracellular calcium. Bottom, A representative digitized contraction profile of an individual LV myocyte exhibiting a homogeneous contraction response during field stimulation at $1 \mathrm{~Hz}$. The digitized contraction profiles were analyzed for specific indices of contractile performance and are summarized in Table I.

length for all myocytes examined is presented in Fig 4. Summary data for isolated LV myocyte contractile function for the total of 804 myocytes is shown in Table I.

After baseline measurements of contractile function, random subgroups of LV myocytes from each patient were exposed to either $12.5-$ or $25-\mathrm{nmol} / \mathrm{L}$ concentrations of isoproterenol. Consistent with $\beta$-adrenergic receptor stimulation, myocyte contractile function significantly increased from baseline values (all $P<.001$ ). The myocyte percent change from baseline after isoproterenol exposure for percent and velocity of shortening for each patient, as well as the overall means, are presented in Fig 5 . In addition, myocyte relengthening velocity increased by $158.5 \%$ above baseline with a $12.5-\mathrm{nmol} / \mathrm{L}$ concentration of isoproterenol and $189.1 \%$ with a 25 nmol/L concentration. Time to peak contraction increased $10.6 \%$ in the $12.5 \mathrm{nmol} / \mathrm{L}$ isoproterenol group and $22.3 \%$ with $25 \mathrm{nmol} / \mathrm{L}$. Values for percent shortening and time to peak contraction were significantly higher in the $25 \mathrm{nmol} / \mathrm{L}$-treated group versus the $12.5 \mathrm{nmol} / \mathrm{L}$-treated group $(P=$ .03 and .004 , respectively). Thus the myocytes isolated from these LV myocardial biopsy specimens retained the capacity to respond to an inotropic stimulus.

Linear and nonlinear regression analysis was performed to determine the relationship between $\mathrm{LV}$ ejection fraction and indices of LV myocyte contractile function. Linear regression coefficients $\left(r^{2}\right)$ for LV ejection fraction relative to percent shortening and velocity of shortening were 0.035 and 0.024 , respectively. Nonlinear, second-order coefficients $\left(r^{2}\right)$ for the same indices were 0.058 and 0.029 . Thus there was no apparent relationship between any of the parameters of myocyte contractility and LV ejection fraction. 

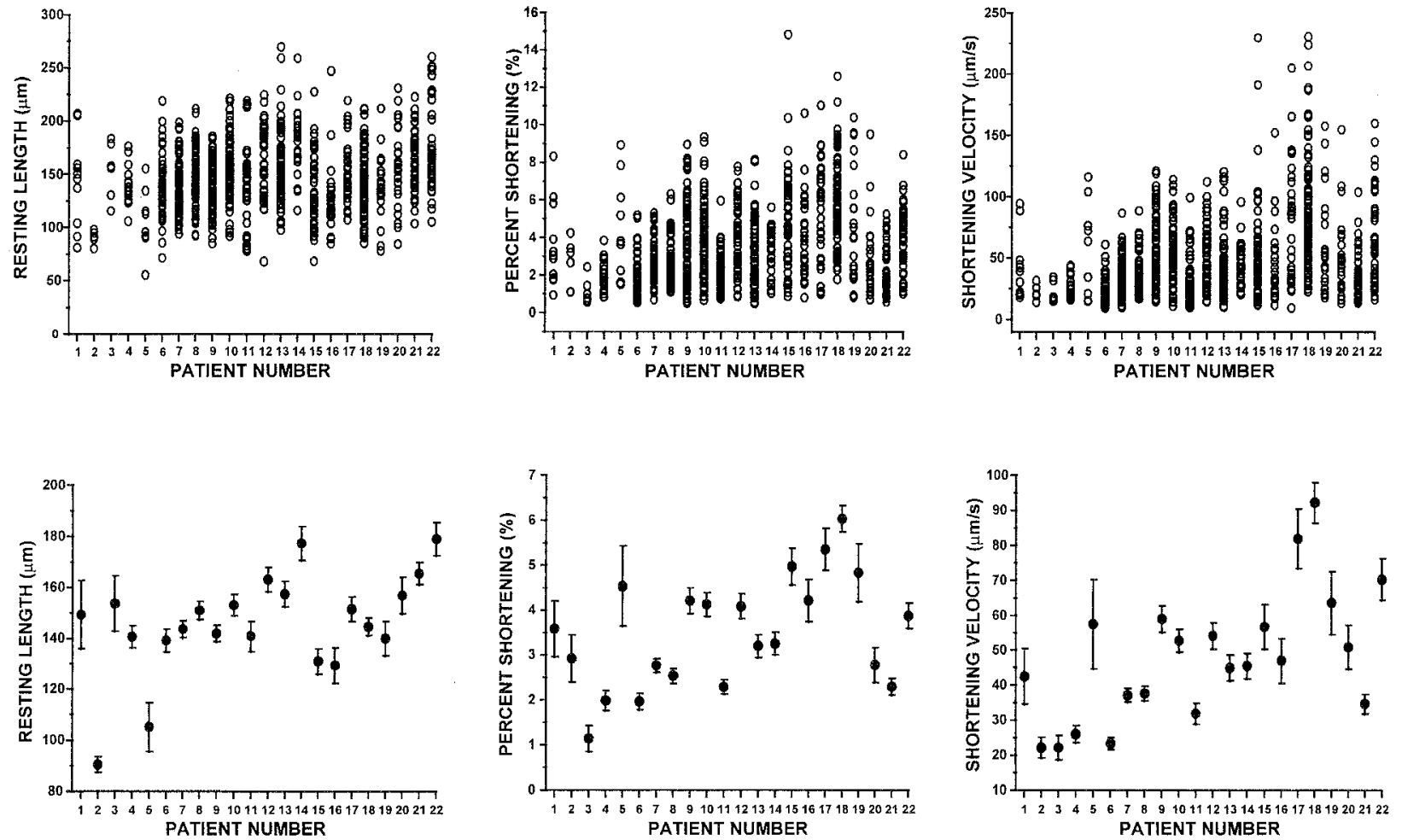

Fig. 3. The actual values of each LV myocyte for each patient are shown in the top graphs for resting length, percent shortening, and shortening velocity, respectively. The bottom graphs demonstrate the mean \pm standard error of the mean values of these indices calculated for the total number of myocytes studied for each patient. The summary data for all of the myocytes included in the study are found in Table I.

\section{Discussion}

Assessment of LV myocardial contractile performance in vivo can be problematic because of dynamic interactions between preload, afterload, and neurohormonal system activational states. Accordingly, methods have been developed in which LV myocytes are isolated from various animal species through coronary perfusion techniques and examined at the cellular level with respect to contractile processes and electrophysiologic events. ${ }^{1-5}$ However, because of the enzymatic digestive methods and amounts of myocardium required for these past studies, evaluation of isolated LV myocyte contractile function in normal human beings has been limited. The goal of the present study was to develop a means to isolate human LV myocytes from small myocardial biopsy specimens collected from patients undergoing elective coronary revascularization procedures and to characterize indices of myocyte contractile performance. The results of the present study demonstrated that a high yield of myocytes could be routinely obtained from human
LV biopsy specimens that were calcium-tolerant, exhibited a homogeneous contraction profile, and maintained the capacity to respond to an inotropic stimulus.

Recent studies have demonstrated that it is possible to isolate myocytes from human myocardium. However, the majority of these studies used myocytes obtained from explanted hearts through perfusion techniques. ${ }^{7-11}$ Moreover, the primary focus of these past studies had been to examine specific aspects of cellular electrophysiology. ${ }^{7,8,11}$ More recently, Peeters and associates ${ }^{12}$ demonstrated the potential for obtaining viable human myocytes from myocardial biopsy specimens that have normal, stable resting membrane potentials. However, it remained unclear whether contractile performance could be consistently measured in myocytes obtained from human LV myocardial biopsy specimens. Harding and colleagues ${ }^{13}$ reported indices of contractile performance in human myocytes isolated by a variety of different methods. In this past report, the average number of myocytes studied from bi- 


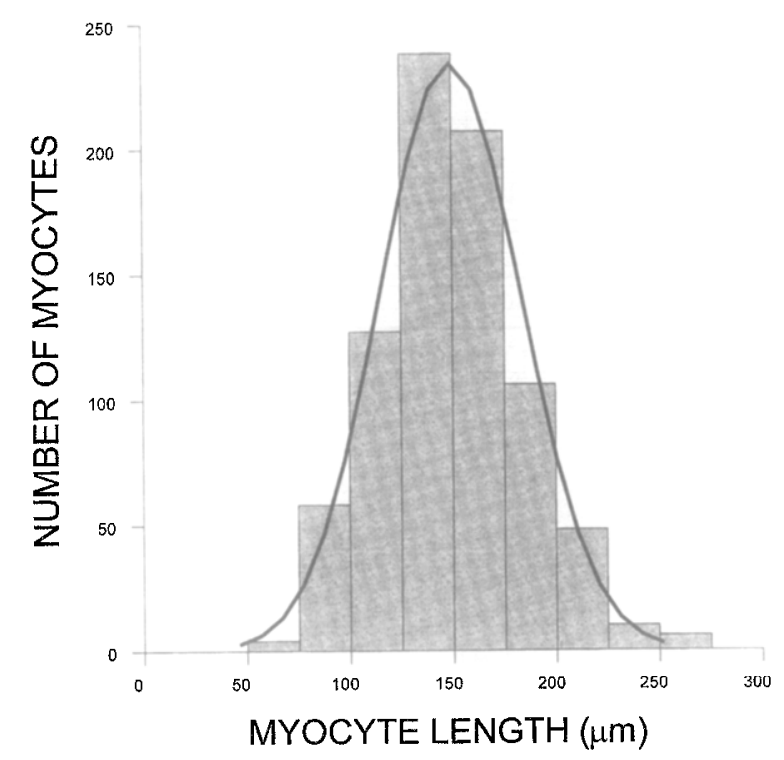

Fig. 4. The distribution of individual myocyte resting lengths for all of the myocytes examined from the LV myocardial biopsy specimens. The mean resting length was $149 \pm 1 \mu \mathrm{m}$. The resting lengths formed a Gaussian distribution as indicated by the solid line.

opsy specimens was less than 2 myocytes per patient, while the present study examined an average of 37 myocytes per patient. Moreover, in the past study by Harding and colleagues, ${ }^{13}$ myocyte contractile function was examined from patients in various stages of heart failure and under a number of different conditions. Thus the present study is the first to characterize myocyte contractile processes and $\beta$-adrenergic responsiveness in a large number of viable myocytes obtained from normal human LV myocardial biopsy specimens.

Past animal studies have indicated that $\beta$-adrenergic receptor system activation increased isolated myocyte contractile function. ${ }^{1,5}, 14,18$ Thus stimulation of the $\beta$-adrenergic receptor system with isoproterenol provides a means to measure the inotropic capacity of the isolated myocyte. Studies of the $\beta$-adrenergic receptor pathway in isolated human myocytes have primarily focused on examining electrophysiologic processes. ${ }^{6,7,9}$ In the present study, isolated human myocyte contractile function was examined with the use of the $\beta$-adrenergic receptor agonist, isoproterenol, at concentrations that had been shown previously to produce a near maximal inotropic response in animal myocyte preparations. ${ }^{17,18,19}$ Use of this $\beta$-adrenergic receptor agonist in the human LV myocyte preparation yielded a
Table I. Isolated human LV myocyte contractile function: Steady-state parameters

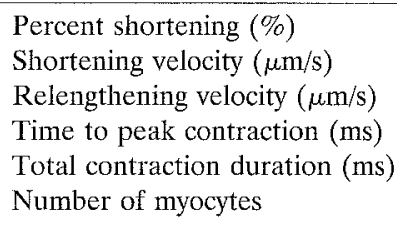

Values presented as mean \pm standard error of the mean.

robust response in contractile performance. Thus the results from this portion of the study demonstrated that myocytes obtained from myocardial biopsy specimens retained the capacity to respond to an inotropic stimulus. On the basis of the apparent dose-dependent response in the present study (25 nmol/L vs $12.5 \mathrm{nmol} / \mathrm{L}$ isoproterenol), future studies using this human isolated myocyte preparation to compute dose-response curves for $\beta$-adrenergic receptor agonists would be warranted.

The myocytes isolated in the present study were determined to be viable on the basis of several criteria as previously described. The concentrations of enzymes (protease, collagenase, and hyaluronidase) used in the isolation procedure were based on previously published studies, $1,7,12,13$ as well as preliminary studies by this laboratory, to maximize cell yields. Past studies from this laboratory have demonstrated that collagenase digestion of isolated LV myocytes using appropriate buffering conditions results in normal resting membrane potentials and no changes in myocyte action potential duration. ${ }^{20}$ Furthermore, this laboratory has demonstrated previously that collagenase digestion of ventricular myocytes does not alter calcium-mediated current or kinetics. ${ }^{20}$ In the present study, collagenase-disassociated myocytes responded to electrical stimulation and to receptor-mediated inotropic modulation. Taken together, these observations suggest that the isolated LV myocyte isolation procedure used herein is not associated with sarcolemmal injury or intrinsic alterations in $\mathrm{Ca}^{++}$homeostasis secondary to the isolation process.

In the isolated myocyte contraction profiles, there appeared to be two phases to the relaxation process. These two phases likely reflect the active relaxation process $\left(\mathrm{Ca}^{++}\right.$sequestration and cross-bridge release) and passive relaxation caused by sustaining forces. ${ }^{21,22}$ Future studies using this isolated system and specific compounds that modulate myocyte relaxation will be necessary to more carefully identify 
PERCENT SHORTENING

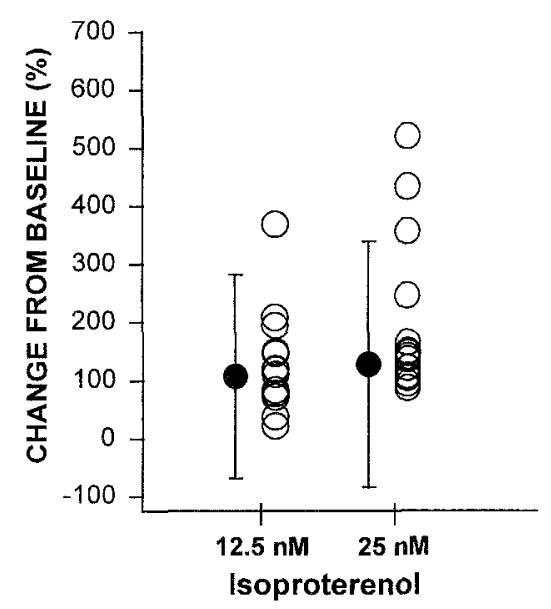

SHORTENING VELOCITY

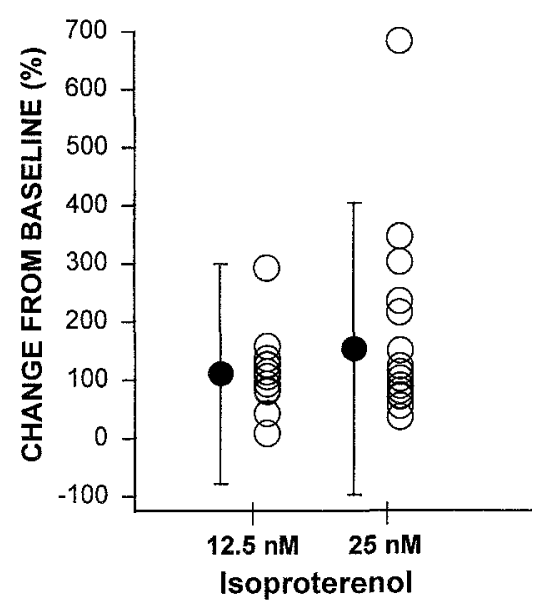

Fig. 5. LV myocyte contractility was measured after $\beta$-adrenergic receptor stimulation with either 12.5 - or $25-\mathrm{nmol} / \mathrm{L}$ concentrations of isoproterenol. The percent change from baseline for percent shortening and shortening velocity is indicated as the response of each subgroup of myocytes for each patient (open circles). The overall response to $\beta$-adrenergic receptor stimulation for all myocytes in each subgroup studied is shown as the mean \pm standard deviation (solid circles). Both percent and velocity of shortening were significantly increased above baseline after $\beta$-adrencrgic receptor stimulation (both $P<.001$ ).

the determinants of the two phases of relaxation observed in the present study.

In past studies in which dog and swine were used, isolated LV myocyte resting length was between 130 and $150 \mu \mathrm{m}^{1,3,4,5,14,17}$ and was very similar to the average human LV myocyte resting length observed in the present study $(\sim 150 \mu \mathrm{m})$. The similarities between $L V$ myocyte resting lengths observed in the present study and in large-animal studies suggest that resting length is conserved among mammalian species. Furthermore, the indices of isolated LV myocyte contractile function observed in the present study were also similar to those obtained from normal isolated $\operatorname{dog}$ and swine myocytes. ${ }^{5,14,17,18}$ Specifically, isolated LV myocyte velocity of shortening has been reported as 50 to $60 \mu \mathrm{m} / \mathrm{s}$, consistent with the results of the present study $(\sim 50 \mu \mathrm{m} / \mathrm{s})$. Percent shortening, reported in healthy animals in the $3.5 \%$ to $5 \%$ range, is also consistent with the observations in the present study $(\sim 3.7 \%)$.

Although in the present study a large number of viable myocytes were obtained from the myocardial biopsy specimens, there are several limitations that should be recognized. First, the myocytes examined in the present study were obtained from a specific region of the anterior $\mathrm{LV}$ myocardium. Whether myocytes from this region exhibit contractile properties representative of myocytes within the entire LV myocardium remains to be determined. Further- more, the LV myocardial biopsy specimens in the present study were obtained from patients with relatively preserved LV ejection fractions undergoing coronary artery bypass operations. Future studies that examine indices of myocyte contractile performance in a wider spectrum of patients with different underlying cardiac disease states would be helpful.

There are limitations of the in vitro system used in the present study, which prevent direct extrapolation to in vivo conditions. First, the isolated myocyte system provides optimal solute diffusion, which does not occur with intact myocardium. Second, this isolated myocyte system removes differences in buffering capacity and extracellular influences that would be operative in pathologic conditions such as hypertrophy and coronary artery disease. Past reports have demonstrated that changes in steadystate isolated myocyte contractile function directly reflect changes in the intrinsic $L V$ contractile performance, as well as the capacity of the LV myocyte to function against a given load. ${ }^{18}$ Using this isolated myocyte system, this laboratory has demonstrated that myocytes respond in a predictable manner to incrementally increased external load. ${ }^{23}$ Thus, although the isolated myocyte function studies described in the present study were performed under equivalent unloaded conditions, it is likely that these findings can be translated into intrinsic myocardial 
contractile capacity. The outcomes of the present study demonstrated that a high yield of isolated myocytes maintaining a normal inotropic response capacity could be reliably obtained from small LV myocardial biopsy specimens taken from patients undergoing cardiac operations. The methods described herein provide a basis by which future studies could investigate intrinsic and extrinsic influences on LV myocyte contractility in human beings.

We express our appreciation to Jeffrey Mandel, Mark Clair, Stephen Krombach, Christopher Kerr, James McElmurray, Terry Heslin, Laura Finklea, and Gregory Austin for tcchnical assistance during the execution of this project.

\section{REFERENCES}

1. Stemmer P, Wisler PL, Watanabe AM. Isolated myocytes in experimental cardiology. In: Fozzard HA, Haber E, Jennings RB, Katz AM, Morgan HE, editors. The heart and cardiovascular system. 2nd ed. New York: Raven Press; 1992. p. 387-404.

2. Bishop SP, Drummond JL. Surface morphology and cell size measurement of isolated rat cardiac myocytes. J Mol Cell Cardiol 1979;11:423-33.

3. Valhouny GV, Tamboli A, Vander Maten M, Albert EN. Morphological and metabolic studies on adult cardiac myocytes. Scan Electron Microsc 1979;3:375-88.

4. Hewett K, Legato MJ, Danilo P Jr, Robinson RB. Isolated myocytes from adult canine left ventricle: $\mathrm{Ca}^{++}$tolerance, electrophysiology, and ultrastructure. Am J Physiol 1983; 245(5 Pt 1):H830-9.

5. Spinale FG, Mukherjee R, Fulbright BM, Hu J, Crawford FA $\mathrm{J}$, Zile MR. Contractile properties of isolated porcine ventricular myocytes. Cardiovasc Res 1993;27:304-11.

6. Gerdes AM, Kriseman J, Bishop SP. Morphometric study of cardiac muscle: the problem of tissue shrinkage. Lab Invest 1982:46:271-4.

7. Hohl CM, Hu B, Wimsatt DK, Fertel RC, Starling RC, Brierley GP, et al. Isolated myocytes from a normal human heart. Prog Clin Biol Res 1990;327:735-42.

8. Li Q, Biagi B, Hohl CM, Starling RC, Stokes B, Altschuld RA. Effects of isoproterenol and caffeine on calcium transients and action potentials in human ventricular cardiomyocytes. Prog Clin Biol Res 1990;327:743-50.

9. Harding SE, Jones SM, O'Gara P, del Monte F, Vescovo G, Poole-Wilson PA. Isolated ventricular myocytes from failing and non-failing human heart: the relation of age and clinical status of patients to isoproterenol response. $\mathrm{J} \mathrm{Mol} \mathrm{Cell}$ Cardiol 1992;24:549-64.

10. Harding SE, Davies CH, Wynne DG, Poole-Wilson PA.
Contractile function and response to agonists in myocytes from failing human heart. Eur Heart J 1994;15(suppl D): 35-6.

11. Altschuld RA, Starling RC, Hamlin RL, Billman GE, Hensley J, Castillo L, et al. Response of failing canine and human heart cells to $\beta$-adrenergic stimulation. Circulation 1995;92: 1612-8.

12. Peeters GA, Sanguinetti MC, Eki Y, Konarzewska H, Renlund DG, Karwande SV, et al. Method for isolation of human ventricular myocytes from single endocardial and epicardial biopsies. Am J Physiol 1995;268:H1757-64.

13. Davies CH, Davia K, Bennett JG, Pepper JR, Poole-Wilson PA, Harding SE. Reduced contraction and altered frequency response of isolated ventricular myocytes from patients with heart failure. Circulation 1995;92:2540-9.

14. Mukherjee R, Crawford FA Jr, Hewett KW, Spinale FG. Cell and sarcomere contractile performance from the same cardiocyte using videomicroscopy. J Appl Physiol 1993; 74:202333.

15. Li T, Sperelakis N, Teneick RE, Solaro RJ. Effects of diacetyl monoxime on cardiac excitation-contraction coupling. J Pharmacol Exp Ther 1985;232:688-95.

16. Mulieri LA, Hasenfuss G, Ittlcman F, Blanchard EM, Alpert NR. Protection of human left ventricular myocardium from cutting injury with 2,3-butanedione monoxime. Circ Res 1989;65:1441-4.

17. Mukherjee R, Spinale FG, Crawlord FA. Measurement of dynamic cellular and sarcomere contractile properties from the same cardiocyte. Proc IEEE Med Biol 1992;7803:392-4.

18. Tsutsui $H$, Spinale FG, Nagatsu $M$, Schmid PG, Ishihara $K$, DeFreyte $G$, et al. The effects of chronic $\beta$-adrenergic blockade on the left ventricular and cardiocyte abnormalities of chronic mitral regurgitation. J Clin Invest 1994;93:2639-48.

19. Tanaka R, Fulbright BM, Mukherjee R, Burchell SA, Crawford FA, Zile MR, et al. The cellular basis for the blunted response to $\beta$-adrenergic stimulation in supraventricular tachycardia-induced cardiomyopathy. J Mol Cell Cardiol 1993; 25:1215-33.

20. Mukherjee R, Hewett KW, Spinale FG. Myocyle electrophysiological properties following the development of supraventricular tachycardia-induced cardiomyopathy. J Mol Cell Cardiol 1995;27:1333-48.

21. Kato S, Koide M, Cooper G IV, Zile MR. Effects of pressure or volume overload hypertrophy on passive stiffness in isolated adult mammalian cardiac muscle cells. Am J Physiol 1996;271:H2575-83.

22. Tagawa $H$, Wang $N$, Narishige $T$, Ingber $D E$, Zile $M R$, Cooper G IV. Cytoskeletal mechanics in pressure overload cardiac hypertrophy. Circ Res 1997:80:281-9.

23. Wang Z, Lam CF, Mukherjee R, Hebbar L, Wang Y, Spinale FG. Relationship between external load and isolated myocyte contractile function with CHF in pigs. Am J Physiol 1997; 273:H183-91. 